CASE REPORT
H. Kato
M. Kanematsu
K. Mizuta
M. Aoki
K. Yamada
H. Yamakawa
T. Iwama
Y. Hirose

\section{Fluid-Fluid Level Formation: A Rare Finding of Extracranial Head and Neck Schwannomas}

SUMMARY: We present 3 cases of extracranial head and neck schwannomas exhibiting fluid-fluid levels. In the described cases, CT and MR imaging showed predominantly cystic components, intermixed with cellular components. Histopathologic examinations of excised specimens revealed hemosiderin deposition, reflecting intratumoral hemorrhages, which was presumably a cause of fluid-fluid levels. Although fluid-fluid levels are nonspecific findings, schwannoma should be considered when radiologic images demonstrate marked cystic formation with fluid-fluid levels in extracranial head and neck tumors.
$\mathbf{S}$ chwannoma is one of the most common soft tissue tumors and constitutes approximately $5 \%$ of all benign neoplasms. The head and neck region is favored, and approximately $25 \%$ to $45 \%$ of benign schwannomas arise in this area. ${ }^{1}$ However, head and neck schwannomas usually occur in the intracranial region and rarely occur extracranially. Although cystic formations are often observed in intracranial and extracranial schwannomas, multicystic formations and intratumoral hemorrhage in schwannomas are relatively rare. Here, we describe the CT and MR imaging findings of 3 patients with extracranial head and neck schwannomas that exhibited fluid-fluid levels, which presumably reflected intratumoral hemorrhage.

\section{Case Reports}

\section{Case 1}

An 85-year-old woman noticed a painless mass on the right side of her neck 4 years before presentation. The mass had grown gradually and had not produced cranial nerve symptoms. Unenhanced CT scan demonstrated a $42 \times 40 \times 72-\mathrm{mm}$, well-demarcated, hypoattenuated lesion with a fluid-fluid level, located in the right middle aspect of the neck (Fig 1A). On unenhanced T1-weighted and T2-weighted MR images, the lesion was found to contain a cystic cavity with a fluid-fluid level and to compress the right common carotid artery anteriorly (Fig 1B). Contrast-enhanced MR images demonstrated a laminated structure, double ringlike enhancement, and slightly mottled enhancement in the outer layer. An open biopsy was performed, and whitish fibrous capsules and internal serous fluid were found. The biopsy also confirmed that the lesion was continuous with the vagus nerve. A histopathologic examination of the biopsy specimen revealed a severely degenerated schwannoma with fibrosis and edema. Hemosiderin deposition was also confirmed, especially within the fibrous tissue.

\section{Case 2}

A 44-year-old man had experienced a pain in the back of his head and neck for 1 year, but the cause of his symptom had not been determined. At presentation, he had lower cranial nerve symptoms,

Received December 16, 2008; accepted December 17.

From the Departments of Radiology (H.K., M.K.), Otolaryngology (K.M., M.A.), Neurosurgery (K.Y., H.Y., T.I.), and Pathology (Y.H.), and High-Level Imaging Diagnosis Center (M.K.), Gifu University School of Medicine, Gifu, Japan.

Please address correspondence to Hiroki Kato, MD, Department of Radiology, Gifu University School of Medicine, 1-1 Yanagido, Gifu 501-1194, Japan; e-mail: hkato@gifu-u.ac.jp DOI 10.3174/ajnr.A1511 namely, paralysis of the soft palate attributed to vagus nerve palsy, and a deviation toward the left side during tongue protrusion because of hypoglossal nerve palsy. Unenhanced CT scan revealed a $44 \times 30 \times$ 44-mm, well-demarcated, hypoattenuated lesion with a hyperattenuated area in a gravity-dependent portion, located in the left parapharyngeal space (Fig 2A). On unenhanced T1-weighted and T2weighted MR images, the lesion was visualized as a unilocular cystic cavity with a fluid-fluid level that caused anterior deviation of the left internal carotid artery (Fig 2B). Contrast-enhanced MR images showed slight enhancement of the cystic walls. The cystic lesion was completely resected and found to have thin cystic walls and to contain a dark reddish fluid. It is unfortunate that, because of adhesions to surrounding tissues, the vagus nerve, which continued with the cystic lesion, could not be preserved. A histopathologic examination of the surgical specimen revealed a schwannoma, predominantly composed of cellular Antoni type A tissue and containing hemosiderin deposition and thrombotic vessels with abnormally thickened walls.

\section{Case 3}

A 59-year-old man had had a painful mass in the right lateral aspect of his neck for 17 years but had refused surgery. Paralysis of his upper and lower extremities and vocal cord palsy had started 6 months before this presentation. Unenhanced CT scan exhibited a $115 \times 70 \times$ 132-mm, well-demarcated, hypoattenuated lesion with a fluid-fluid level, located in the right aspect of the neck (Fig $3 A$ ). The lesion had also invaded the posterior cranial fossa and eroded the skull base. Unenhanced T1-weighted and T2-weighted MR images showed multicystic formation with a fluid-fluid level and an anterior deviation of the right internal and external carotid arteries because of compression by the lesion (Fig 3B). Contrast-enhanced MR images showed heterogeneous, reticular enhancement of the cystic walls and the septa. The lesion was completely resected by 2 -stage surgery and was found to have thin cystic walls and to contain serous fluid. Because of the size of the tumor, its originated nerve was not determined. A histopathologic examination of the surgical specimen revealed a schwannoma occupied with cellular Antoni type A tissue, containing massive amounts of hemosiderin, thrombotic vessels, and abnormal vessel proliferation.

\section{Discussion}

The histologic finding of schwannoma typically displays a biphasic pattern with areas of highly ordered cellularity (Antoni type A) and hypocellular regions dominated by a highly myxoid matrix (Antoni type B). Furthermore, large or rapidly growing schwannomas commonly undergo degenerative 

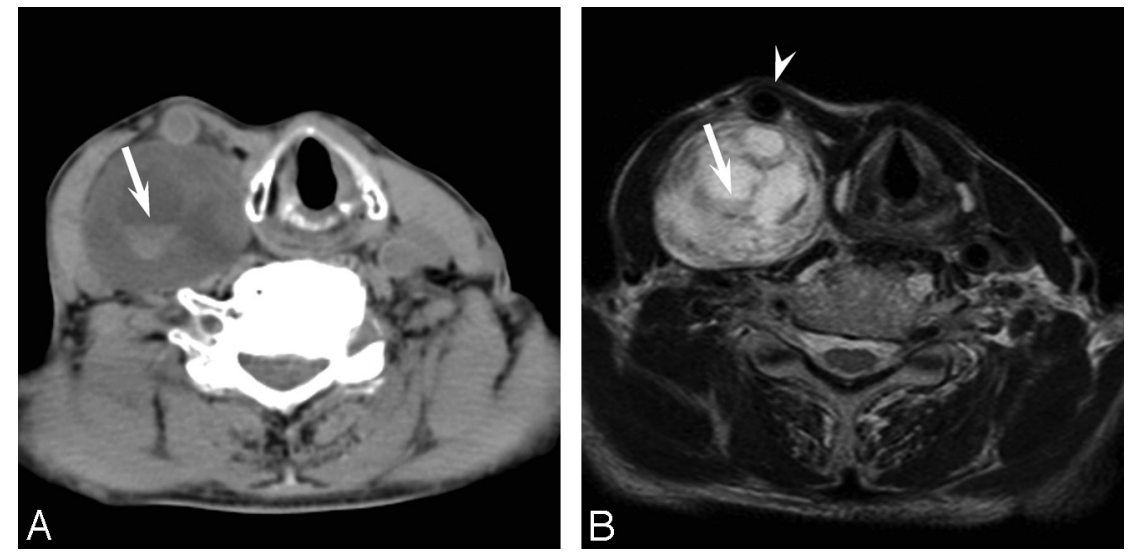

Fig 1. Case 1. An 85-year-old woman with a vagus nerve schwannoma in the right neck. A, Unenhanced CT scan shows a well-demarcated, hypoattenuated lesion, with a fluid-fluid level (arrow). B, Axial T2-weighted fast spin-echo MR image (TR, $4102 \mathrm{~ms}$; TE, $90 \mathrm{~ms}$ ) shows a cystic tumor with a fluid-fluid level (arrow) and a deviation of the right common carotid artery (arrowhead).
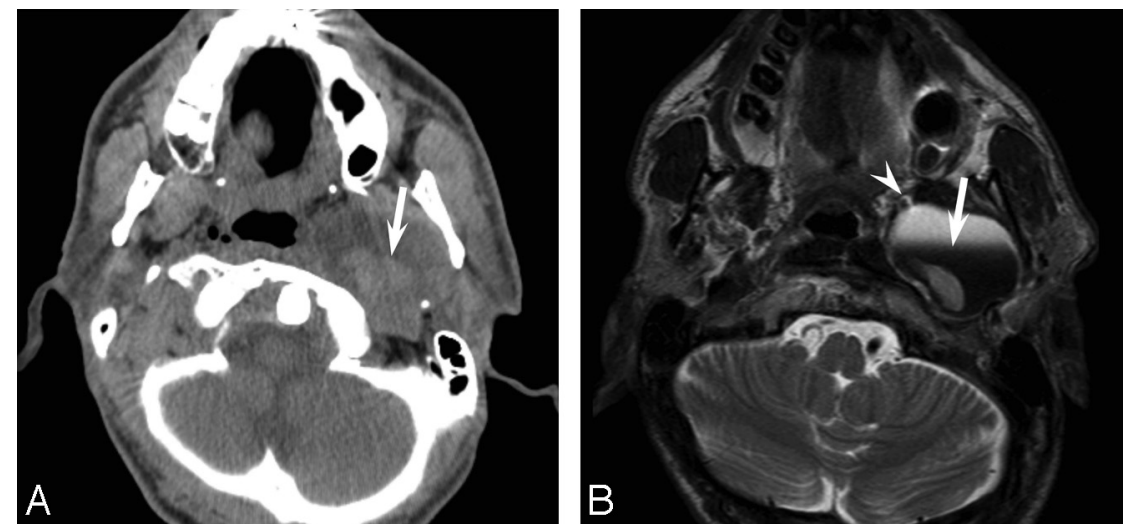

Fig 2. Case 2. A 44-year-old man with a vagus nerve schwannoma in the left parapharyngeal space. A, Unenhanced CT scan shows a well-demarcated, hypoattenuated lesion, with a fluid-fluid level (arrow). B, Axial T2-weighted fast spin-echo MR image (TR, $5710 \mathrm{~ms}$; TE, $90 \mathrm{~ms}$ ) shows a unilocular cystic tumor with a fluid-fluid level (arrow) and a deviation of the left internal carotid artery (arrowhead).
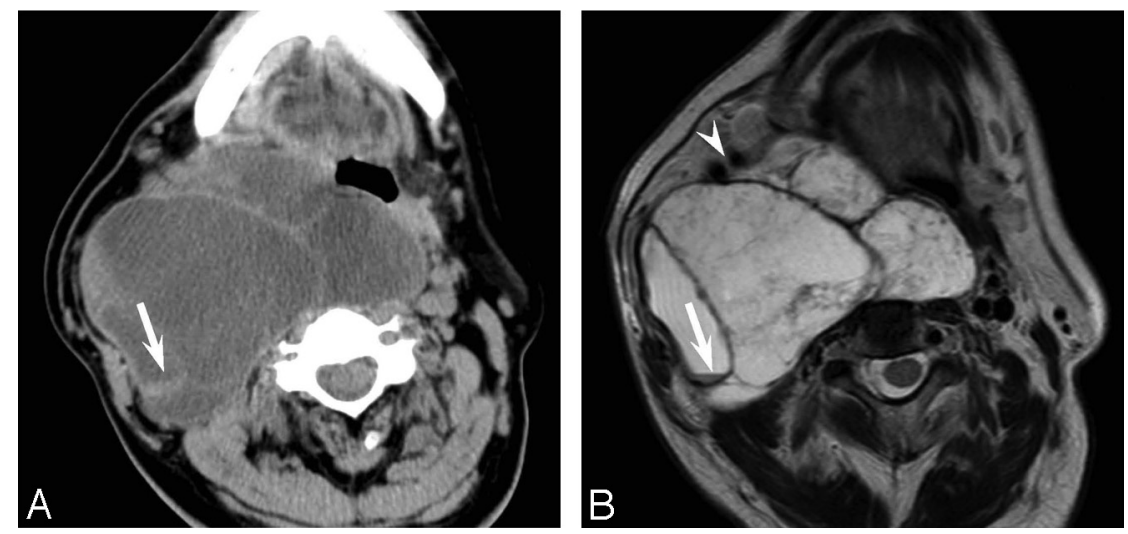

Fig 3. Case 3. A 59-year-old man with a large schwannoma in the right neck, extending to the posterior cranial fossa. A, Unenhanced CT scan shows a well-demarcated, hypoattenuated lesion, with a fluid-fluid level (arrow). B. Axial T2-weighted fast spin-echo MR image (TR, $4102 \mathrm{~ms}$; TE, $90 \mathrm{~ms}$ ) shows a multicystic tumor with a fluid-fluid level (arrow) and a deviation of the right internal and external carotid arteries (arrowhead).

changes, which include cyst formation, calcification, hemorrhage, and fibrosis, and 1 rare schwannoma variant (often referred to ancient schwannoma) is characterized by remarkable degeneration and diffuse hypocellular areas. These degenerative changes are thought to be the result of long-term progression. The CT findings of schwannomas have been described as well-circumscribed, heterogeneous, and hypoattenuated. As was observed in our cases, ancient schwannomas have been reported to show enhancement in peridegenerative areas and at fibrous capsules on contrast-enhanced CT and MR images. ${ }^{2}$

Some authors have proposed that a confluence of mucinous and microcystic areas in Antoni type B tissue accounts for most large cysts produced in schwannomas. ${ }^{3}$ Antoni type $B$ tissue is believed to produce a myxomatous material in small cystic areas that coalesces into larger cysts and compresses surrounding Antoni type A tissue. However, the relationship be- 
tween cystic formation and the distribution of Antoni cell types is not always observed. ${ }^{4}$ In cases 2 and 3, cellular Antoni type A tissue occupied most of the tumor.

On the other hand, microhemorrhage is also considered to be a mechanism of cystic formation in schwannomas, and hemosiderin-laden macrophages, hemosiderin deposits, and thrombotic vessels are more frequently observed in cystic vestibular schwannomas than in solid vestibular schwannomas. ${ }^{4}$ Hemosiderin deposition was obvious in all of our cases, and thrombotic vessels and abnormal vessel proliferation were observed in cases 2 and 3. Furthermore, xanthochromic fluid within the cysts was found during surgery, which supports the above hypothesis.

Fluid-fluid levels are nonspecific imaging findings that may be observed in a variety of neoplastic, inflammatory, traumatic, and other miscellaneous pathologic conditions. Neoplastic fluid-fluid levels are observed in $2.7 \%$ of bone tumors and in $2.9 \%$ of soft tissue tumors. ${ }^{5}$ However, fluid-fluid levels have been reported in various tumors, regardless of benignity or malignancy. ${ }^{6}$ In soft tissue tumors, fluid-fluid levels have been reported in schwannomas, cavernous hemangiomas, and sarcomas (eg, in synovial sarcoma and angiosarcoma, and in malignant fibrous histiocytosis). ${ }^{5,6}$ On the basis of viscosity and protein content measurements after hemorrhage or necrosis, fluid-fluid levels are caused by the separation of unclotted blood as opposed to serous (interstitial) fluid. ${ }^{1}$

Although fluid-fluid level formation within schwannomas is a somewhat rare imaging finding, several reports have mentioned this finding in the head and neck region. However, most of these reports concern schwannomas, especially vestibular schwannomas, with fluid-fluid levels in the intracranial region, and relatively few reports have addressed the extracranial region. MR imaging is more sensitive than CT to detect fluid-fluid levels because of its better contrast resolution and sensitivity for subacute and chronic hemorrhage. In our 3 extracranial head and neck schwannoma cases, both CT and MR imaging demonstrated fluid-fluid levels.

In conclusion, although fluid-fluid level formation is a nonspecific imaging finding that may be encountered in miscellaneous pathologic conditions, its presence, like anterior deviation of the carotid artery, undoubtedly narrows the differential diagnoses. Accordingly, schwannoma should be considered when radiologic images demonstrate marked cystic formation with fluid-fluid levels in an extracranial head and neck tumor.

\section{References}

1. Catalano P, Fang-Hui E, Som PM. Fluid-fluid levels in benign neurogenic tumors. AJNR Am J Neuroradiol 1997;18:385-87

2. Isobe $\mathrm{K}$, Shimizu $\mathrm{T}$, Akahane $\mathrm{T}$, et al. Imaging of ancient schwannoma. $A J R$ Am J Roentgenol 2004;183:331-36

3. Wallace CJ, Fong TC, Auer RN. Cystic intracranial schwannoma. Can Assoc Radiol J 1993;44:453-59

4. Park CK, Kim DC, Park SH, et al. Microhemorrhage, a possible mechanism for cyst formation in vestibular schwannomas. J Neurosurg 2006;105:576-80

5. Van Dyck P, Vanhoenacker FM, Vogel J, et al. Prevalence, extension and characteristics of fluid-fluid levels in bone and soft tissue tumors. Eur Radiol 2006;16:2644-51

6. Tsai JC, Dalinka MK, Fallon MD, et al. Fluid-fluid level: a nonspecific finding in tumors of bone and soft tissue. Radiology 1990;175:779-82 